\title{
Revision der Mehrwertsteuer: Vereinfachung ja, aber nicht zu jedem Preis
}

Die Revision der Mehrwertsteuer (MWST) beschäftigt die Ärzteschaft nun bereits seit mehr als zwei Jahren: Im April 2006 reichte die FMH zuhanden der Expertenkommission «Spori» eine Stellungnahme ein. Im Juli 2007 folgte die Vernehmlassung an das Eidgenössische Finanzdepartement. Und vor wenigen Tagen nahm die FMH an einem Hearing der Kommission für Wirtschaft und Arbeit des Nationalrats teil, an dem die bundesrätliche Botschaft zur MWST-Revision behandelt wurde. Teil A dieser Botschaft enthält über 50 Massnahmen für ein vollständig überarbeitetes Mehrwertsteuergesetz. Teil B sieht vor, einen einheitlichen Steuersatz von 6,1 Prozent einzuführen und die meisten der heute bestehenden Steuerausnahmen abzuschaffen.

Die Meinung der FMH zur bundesrätlichen Botschaft ist klar: Aus Sicht der Ärzteschaft ist es notwendig, das MWST-Verfahren zu vereinfachen. Doch die «Ausnahme Gesundheitswesen»- eigentlich handelt es sich um eine ganze Reihe von Ausnahmen im Bereich des Gesundheitsund Sozialwesens, die im geltenden Artikel 18 MWSTG verankert sind - muss beibehalten werden. Zahlreiche sozialpolitische und volkswirtschaftliche Argumente sprechen gegen die Abschaffung:

- Werden die Heilbehandlungen neu der MWST unterstellt, führt dies im Gesundheitswesen zu einem Kostenschub in dreistelliger Millionenhöhe. Die Krankenkassenprämien würden zusätzlich um über 4 Prozent steigen, was ein sozialpolitisches Korrektiv für einkommensschwache Haushalte notwendig macht.

- Bei der vorgeschlagenen Aufhebung der meisten Steuerausnahmen ist mit rund 30000 neuen Steuerpflichtigen zu rechnen. Für diese und deren Verbände, aber auch für die Verwaltung entsteht ein erheblicher administrativer Mehraufwand.

- Im Vergleich zur EU, in der die Heilbehandlungen im Bereich der Humanmedizin von der MWST ausgenommen sind, führt die Abschaffung der «Ausnahme Gesundheitswesen» zu einem grossen Wettbewerbsnachteil für den Wirtschaftsstandort Schweiz.
- Die geplante Einlageentsteuerung, die für die neuen Steuerpflichtigen den Nachteil ausgleichen soll, dass sie auf bereits getätigten Investitionen keinen Vorsteuerabzug geltend machen konnten, generiert für den Bund hohe Kosten.

- Es ist widersprüchlich, den Berufsverbänden gesetzliche Aufgaben zu erteilen, die Finanzierung dieser Aufgaben (via die Mitgliederbeiträge) aber mit MWST zu belasten.

- Praxisgemeinschaften werden durch die geplante Revision ebenfalls benachteiligt. So ist vorgesehen, jene Ausnahmeregelung zu streichen, die zum Beispiel das interne Weiterverteilen von gemeinsam gekauften Medikamenten von der MWST ausnimmt.

All diese Argumente überwiegen die Vorteile der angestrebten Vereinfachung. Der Widerstand gegen die Aufhebung der «Ausnahme Gesundheitswesen» ist wohl auch deshalb so breit abgestützt. Die Gesundheitsdirektoren der Kantone haben sich ebenso deutlich gegen die Vorlage ausgesprochen wie etwa der Spitalverband H+ oder santésuisse für die Krankenversicherer. Alle warnen insbesondere vor dem massiven Kostenschub im Gesundheitswesen und den unweigerlich steigenden Krankenkassenprämien. Das Dossier liegt nun bei der Kommission für Wirtschaft und Arbeit des Nationalrates. Nach dem Hearing, an dem die FMH ihre Position nochmals deutlich gemacht hat, wird sich die Kommission in den kommenden Sitzungen inhaltlich mit der Vorlage befassen. Wir werden den Fortgang der Debatte aufmerksam verfolgen und uns weiterhin nachdrücklich dafür einsetzen, dass es keinen mehrwertsteuerbedingten Teuerungsschub im Gesundheitswesen gibt.

Dr. med. Jacques de Haller, Präsident der FMH 